\title{
Size Tunable Synthesis and Surface Chemistry of Metastable $\mathrm{TiO}_{2}$-Bronze Nanocrystals
}

Jonas Billet, ${ }^{1}$ Wouter Dujardin, ${ }^{1}$ Katrien De Keukeleere, ${ }^{1}$ Klaartje De Buysser, ${ }^{1}$ Jonathan De Roo, ${ }^{1,2 *}$ Isabel Van Driessche ${ }^{1 *}$

${ }^{1}$ Ghent University, Department of Chemistry, Krijgslaan 281 S3, 9000 Gent, Belgium

${ }^{2}$ Columbia University, Department of Chemistry, 3000 Broadway, New York, NY 10027, USA

Corresponding authors:

Jonathan De Roo, Jonathan.DeRoo@ugent.be

Isabel Van Driessche, Isabel.VanDriessche@ugent.be

\begin{abstract}
The metastable titania phase, bronze, has great potential as photo-catalyst or as anode material in Li-ion batteries. Here, we report the first synthesis of colloidally stable, size-tunable $\mathrm{TiO}_{2}$-bronze $\left(\mathrm{TiO}_{2}-\mathrm{B}\right)$ nanocrystals, via a hydrothermal process. By employing Definitive Screening Design, the experimental parameters affecting the size and agglomeration of the nanocrystals are identified. The size is mostly determined by the reaction temperature, resulting in $3-8 \mathrm{~nm}$ NCs in the range of $130{ }^{\circ} \mathrm{C}-180{ }^{\circ} \mathrm{C}$. To avoid irreversible aggregation, short reaction times are desired and in this respect microwave heating proved essential due to its fast heating and cooling rates. The resulting nanocrystals are de-aggregated and stabilized in polar solvents using either positive or negative surface charges. In nonpolar solvents, steric stabilization is provided by long chain amines and carboxylic acids. Furthermore, we study this peculiar post-synthetic surface modification through solution ${ }^{1} \mathrm{H}$ NMR and elemental analysis. Surprisingly, we find ion-pairs of alkylammonium carboxylates bound to the surface, contrasting with earlier reports on carboxylic acid stabilized metal oxide nanocrystals. To rationalize this, a general framework of acid/base chemistry with metal oxide nanocrystals is constructed and discussed.
\end{abstract}




\section{Introduction}

Titanium dioxide $\left(\mathrm{TiO}_{2}\right)$ is abundant, non-toxic, and thermally, physically and chemically stable. It is employed in diverse applications ranging from white pigments in food or cosmetics, to (photo) catalysis, gas sensing, fuel cells and batteries. ${ }^{1-4}$ In contrast to the three well-known crystal polymorphs of $\mathrm{TiO}_{2}$ (anatase, rutile, brookite), the metastable, monoclinic bronze phase; $\mathrm{TiO}_{2}-\mathrm{B}$, is far less studied. Nonetheless, interest in this crystal phase has grown significantly in the last decade due to its potential as photocatalyst, ${ }^{5-7}$ electrochromic layer ${ }^{8}$ and as anode material in Li-ion batteries. ${ }^{9-15}$ However, $\mathrm{TiO}_{2}-\mathrm{B}$ suffers from both low electronic and ionic diffusion rates. ${ }^{9}{ }^{16}$ Hence the general strategy of reducing the dimensions to the nanoscale, in order to reduce the diffusion length. ${ }^{17}$

There are three main synthetic strategies for nanoscale $\mathrm{TiO}_{2}-\mathrm{B}$. In the first approach, anatase or rutile $\mathrm{TiO}_{2}$ is dissolved with strong base and the resulting layered titanates, $\mathrm{H}_{2} \mathrm{Ti}_{4} \mathrm{O}_{9}$, are calcined at $500{ }^{\circ} \mathrm{C}$ to form the Bronze phase. ${ }^{18}$ This method has been adapted for the synthesis of nanowires, ${ }^{19,}{ }^{20}$ nanotubes $^{21}$ and nanorings. ${ }^{12}$ In a second approach, a titanium precursor $\left(\mathrm{TiCl}_{3}, \mathrm{TiCl}_{4}, \mathrm{Ti}(\mathrm{OEt})_{4}\right.$ or $\left.\mathrm{Ti}(\mathrm{O} i \mathrm{Pr})_{4}\right)$ is solvothermally reacted in an organic solvent (ethylene glycol or 1-octadecene) under acidic or basic conditions, leading to $\mathrm{TiO}_{2}-\mathrm{B}$ nanosheets, ${ }^{7}, 13,22$ nanowires, ${ }^{23}$ nanorods $^{24}$ or meso-structures. ${ }^{25,} 26$ Both these methods lead to aggregated nanoparticles with phase impurities (anatase/rutile), are time consuming and are unable to synthesize nanocrystals smaller than $10 \mathrm{~nm}$ in diameter. As a third strategy, Kobayashi et al. developed an one-step hydrothermal procedure for $\mathrm{TiO}_{2}-\mathrm{B}$ nanocrystals $(\mathrm{NCs}) .{ }^{27}$ In this straightforward synthesis, an aqueous solution of a titanium glycolic acid complex is hydrothermally treated in a pressure bomb at $160^{\circ} \mathrm{C}$ for $24 \mathrm{~h}$. Although the primary nanocrystals are small $(6 \mathrm{~nm})$, highly crystalline and pure $\mathrm{TiO}_{2}-\mathrm{B}$, they are extensively agglomerated into secondary particles of $0.3-3 \mu \mathrm{m} .^{11,28,29}$

To optimize the performance of photocatalytic processes or Li-ion batteries, colloidally stable NCs with adjustable surface chemistry and tunable size are required. Small NCs are compatible with small electron/ion diffusion coefficients, ${ }^{9}$ feature larger surface areas and can be assembled into frameworks at high loading. ${ }^{30}$ However, larger particles suppress undesired surface effects, ${ }^{11}$ reduce the amount of electron-hopping sites ${ }^{31}$ and feature a higher crystallinity. Therefore, every application features an optimum nanocrystal size corresponding to maximum performance. However, such a size dependent analysis is only possible on uniform, aggregation-free samples. The same conditions apply for the general patterning, ${ }^{8,32}$ assembly $^{33}$ or deposition ${ }^{34}$ of NCs. Typically, to obtain colloidally stabilized NCs, organic 
surfactants are either present during the synthesis ${ }^{35,36}$ or are added post-synthesis. ${ }^{37-39}$ In both cases, the nature and coordination of the ligands to the surface, i.e., the surface chemistry, ${ }^{34}$ is key to the colloidal stability. In addition, the surface chemistry is often the determining factor in nano-catalysis, ${ }^{40}$ photoluminescence, ${ }^{41,}{ }^{42}$, photovoltaics,${ }^{43}$ electrochromics ${ }^{44}$ and thermoelectrics. ${ }^{45}$ Unfortunately, none of the current synthetic protocols delivers colloidally stable $\mathrm{TiO}_{2}-\mathrm{B}$ nanocrystals with tunable size or surface chemistry.

Given the phase purity and the small NC size, we take the hydrothermal process of Kobayashi et. al. as a starting point and redesign it into the first agglomeration-free synthesis of monodisperse $\mathrm{TiO}_{2}-\mathrm{B}$ NCs, size controlled from 3 to $8 \mathrm{~nm}$. In an innovative and systematic approach, Definitive Screening Design (DSD) is used to identify the governing parameters. ${ }^{46}$, 47 To reduce irreversible aggregation, it is imperative to replace the slow traditional heating with fast microwave heating (5-45 min). Furthermore, by adjusting the surface chemistry, we stabilize $\mathrm{TiO}_{2}$-B NCs in both polar and nonpolar media. In polar media (water, $\mathrm{MeOH}, \mathrm{EtOH}$, DMF), the NCs feature a negative zeta potential due to the basic environment generated by bases like triethylamine (TEA) or 1,8-diazobicyclo[5.4.0]undec-7-ene (DBU). In nonpolar media $\left(\mathrm{CHCl}_{3}\right.$ or toluene), oleylamine (OLAm) also removes acidic surface protons and stabilizes the nanocrystals to a limited extent. Furthermore, oleic acid (OLAc) removes the surface bound sulfate groups and completes the de-aggregation. Finally, the NCs could also be stabilized in DMF with a positive surface charge by removing the organic ligands with $\mathrm{BF}_{3}: \mathrm{Et}_{2} \mathrm{O}$. Since both the size and surface chemistry of these $\mathrm{TiO}_{2}-\mathrm{B}$ NCs can be precisely tuned, they are the ideal building blocks for all applications of $\mathrm{TiO}_{2}-\mathrm{B}$.

\section{Experimental Section}

General considerations. Titanium (Sigma - 99.7\%), hydrogen peroxide (Honeywell - 35\%), ammonia (Fisher -28\%), glycolic acid (Sigma - 99\%), sulfuric acid (Roth - 96\%), triethylamine (Sigma - 99.5\%), oleylamine (Sigma - 98\%), oleic acid (Sigma - 99\%), methanol (Fiers - 99.8\%), ethanol (PanReac - 99.8\%), chloroform (Carlo Erba - 99.9\%), 1,8diazabicyclo[5.4.0] undec-7-ene (Fluka - 98\%), deuterium oxide (Euriso-top - 99.9\%), octylamine (Sigma - 99\%), dodecanoic acid (Sigma - 98\%), chloroform-d (Euriso-top 99.8\%), hexane (Sigma - $\geq 95 \%$ ), toluene (Sigma - 99.8\%), BF 3 :Et $2 \mathrm{O}$ (Acros - 48\%), dimethylformamide (Sigma - 99.8\%) and acetone (Fiers - 99.5\%) were used without purification. 
Nanocrystal synthesis. $\mathrm{TiO}_{2}$ - $\mathrm{B}$ NCs were synthesized in a microwave setup, with the titanium precursor synthesized according to Kobayashi et. al. ${ }^{27}$ In a reference synthesis, titanium powder $(0.047 \mathrm{~g}, 1.0 \mathrm{mmol})$ is dissolved overnight in a mixture of $\mathrm{H}_{2} \mathrm{O}_{2}$ solution $(4 \mathrm{~mL}, 60 \mathrm{mmol})$ and ammonia solution $(1 \mathrm{~mL}, 12 \mathrm{mmol})$ in water) resulting in a clear yellow solution. Next, 0.114 $\mathrm{g}(1.5 \mathrm{mmol})$ of glycolic acid is added, and this mixture is heated to $80^{\circ} \mathrm{C}$ until a gel-like yellow precursor remains. This gel is dissolved in $2 \mathrm{~mL} \mathrm{H}_{2} \mathrm{O}$ and $0.13 \mathrm{~mL}$ sulfuric acid (2.3 mmol). The total volume of the precursor is adjusted to $4 \mathrm{~mL}$ with $\mathrm{H}_{2} \mathrm{O}$, and added to a $10 \mathrm{~mL}$ microwave vial. This solution is heated for $30 \mathrm{~min}$ at $160^{\circ} \mathrm{C}$, yielding a white finely precipitated suspension of $\mathrm{TiO}_{2}-\mathrm{B}$ NCs. The synthesis was performed with a CEM Discover microwave set up equipped with auto sampler and IR temperature detection. For a standard $160{ }^{\circ} \mathrm{C}-30 \mathrm{~min}$ reaction a typical pressure/power/temperature profile is given in Fig S1.

Surface modification. The homogenized reaction mixture $(1 \mathrm{~mL}, 0.25 \mathrm{mmol} \mathrm{Ti})$ was centrifuged at $4000 \mathrm{rpm}$ for $1 \mathrm{~min}$. The white precipitate was washed 3 times with $\mathrm{H}_{2} \mathrm{O}$ and centrifuged at $4000 \mathrm{rpm}$ for $1 \mathrm{~min}$. To this white solid, oleylamine (OLAm, $100 \mu \mathrm{L}, 0.3 \mathrm{mmol}$ ) was added and stirred for $30 \mathrm{~min}$. Subsequently, chloroform (1 mL) and oleic acid (OLAc, 100 $\mu \mathrm{L}, 0.32 \mathrm{mmol}$ ). This clear suspension is precipitated with $2 \mathrm{ml}$ acetone and re-suspended in chloroform. The ligands could be removed and stabilized in DMF with a positive surface charge using the procedure described in Doris et. al. ${ }^{48}$ In particular, $\mathrm{TiO}_{2}-\mathrm{B}$ NCs $(0.25 \mathrm{mmol} \mathrm{Ti})$ stabilized with OLAm/OLAc were suspended in $0.5 \mathrm{ml}$ of $\mathrm{CHCl}_{3}$ and $0.5 \mathrm{ml}$ hexane. To this suspension, $20 \mu \mathrm{BF}_{3}: \mathrm{Et}_{2} \mathrm{O}$ in $1 \mathrm{ml}$ DMF was added and the mixture was rigorously stirred. After 5 minutes a white precipitate appeared, which was centrifuged at $4000 \mathrm{rpm}$ for $1 \mathrm{~min}$. The white precipitate could be redispersed in DMF $(1 \mathrm{ml})$ and precipitated with toluene. To stabilize the nanocrystals polar solvents with a negative surface charge, the post-synthetic white precipitate ( $1 \mathrm{~mL}$ of reaction mixture) was, after washing with $\mathrm{H}_{2} \mathrm{O}$, air dried and to this solid triethylamine $(15 \mu \mathrm{L}, 0.07 \mathrm{mmol})$ was added and stirred. To this clear solution, $1 \mathrm{~mL}$ of $\mathrm{H}_{2} \mathrm{O}$ (or $\mathrm{MeOH}, \mathrm{EtOH}$, etc.) was added, subsequently giving a clear colorless suspension.

\section{DSD experiment}

The design was constructed around four factors $(\mathrm{k}=4)$, the amount of glycolic acid $\left(\chi_{1}\right)$, sulfuric acid $\left(\chi_{2}\right)$, the microwave temperature $\left(\chi_{3}\right)$ and the microwave time $\left(\chi_{4}\right)$. The factor levels (minimum, center, maximum) were chosen corresponding with a preceding design study, which can be found in the supporting info. A four factor design needs 13 experiments to identify the factors causing a significant nonlinear effect. ${ }^{46}$ Two responses are examined: the nanocrystal size $\left(\mathrm{Y}_{1}\right)$ and the agglomeration $\left(\mathrm{Y}_{2}\right)$, while the crystal phase is monitored to be the desired 
Bronze phase. The nanocrystal size is determined by calculating the mean size of an ensemble of +100 nanocrystals selected from TEM images. The agglomeration $\left(\mathrm{Y}_{2}\right)$ was set to be the Zaverage value from DLS, determined from 8x diluted OLAm/OLAc stabilized $\mathrm{NCs}$ in $\mathrm{CHCl}_{3}$. For the analysis of the responses, the statistical software package $\mathrm{JMP}^{\circledR}$ was used.

\section{Characterization}

A Malvern Nano ZS was used in back-scattering mode $\left(173^{\circ}\right)$ for dynamic light scattering (DLS) and zeta-potential measurements. Zeta-potentials were measured at $40 \mathrm{~V}$ in monomodal mode. Transmission electron microscopy (TEM) images were taken on a JEOL JEM-2000FS TEM with Cs corrector. Powder X-ray diffraction (PXRD) patters of the adsorbents were collected on a Thermo Scientific ARL XTra diffractometer, operated at $40 \mathrm{kV}, 40 \mathrm{~mA}$ using $\mathrm{Cu} \mathrm{K \alpha}$ radiation with $0.15418 \mathrm{~nm}$ wavelength. Nuclear magnetic resonance (NMR) measurements were recorded on a Bruker Avance III spectrometer operating at a ${ }^{1} \mathrm{H}$ frequency of $500.13 \mathrm{MHz}$ and equipped with a BBI-Z probe. The sample temperature was set at $298.2 \mathrm{~K}$. One-dimensional (1D) 1H, HSQC, HMBC, COSY and 2D NOESY (nuclear Overhauser effect spectroscopy) spectra were acquired using standard pulse sequences from the Bruker library. NOESY mixing time was set to $300 \mathrm{~ms}$, and 2048 data points in the direct dimension for 512 data points in the indirect dimension were typically samples, with the spectral width set to 11.5 ppm. Diffusion measurements (2D DOSY) were performed using a double-stimulated echo sequence for convection compensation and with monopolar gradient pulses.

\section{Results and Discussion}

Microwave versus traditional heating. A titanium-peroxo compound is synthesized according to Kobayashi et al. ${ }^{27,49}$ Subsequently, a complex with glycolic acid is formed and the $\mathrm{pH}$ is adjusted with sulfuric acid (Scheme 1). The complex is decomposed into $\mathrm{TiO}_{2}-\mathrm{B} \mathrm{NCs}$ in a hydrothermal synthesis, executed either in a specialized microwave setup (with a glass vessel) or in a traditional oven (with a stainless-steel bomb).

$$
\left(\mathrm{NH}_{4}\right)\left[\mathrm{Ti}(\mathrm{OH})_{3} \mathrm{O}_{2}\right]+{\underset{\mathrm{OH}}{\mathrm{OH}}}_{\mathrm{O}}^{\mathrm{O}}+\mathrm{H}_{2} \mathrm{SO}_{4} \underset{\text { water }}{\stackrel{130-180^{\circ} \mathrm{C}}{\longrightarrow}} \mathrm{TiO}_{2}-\mathrm{B}
$$

Scheme 1. Synthetic approach to bronze $\mathrm{TiO}_{2}$ nanocrystals.

Microwave heating results in a nanocrystal powder that can be de-aggregated into individual nanocrystals (Figure 1A) by the addition of a specific surfactant combination; a 1:1 ratio of oleylamine and oleic acid. This post-synthetic treatment renders the particles dispersible in nonpolar solvents such as chloroform and toluene. Although at first sight this surface 
modification seems identical to the de-aggregation of $\mathrm{ZrO}_{2}$ and $\mathrm{HfO}_{2} \mathrm{NCs}$ synthesized in benzyl alcohol, ${ }^{39,50}$ the final surface chemistry is very different (vide infra). In contrast to the microwave synthesis, the NCs synthesized in a traditional oven are irreversibly agglomerated into 0.3 - $3 \mu \mathrm{m}$ secondary particles and the same post-synthetic surface modification could not fully disperse or de-aggregate the NCs (Figure 1B). Nevertheless, the size of the primary particles $(5.5 \mathrm{~nm})$ is the same, irrespective of the heating method. In addition, both the traditional oven and the microwave furnace provides phase-pure bronze $\mathrm{TiO}_{2}$ according to the XRD diffractogram (Figure 1C) with crystallite domains of $4.8 \mathrm{~nm}$.

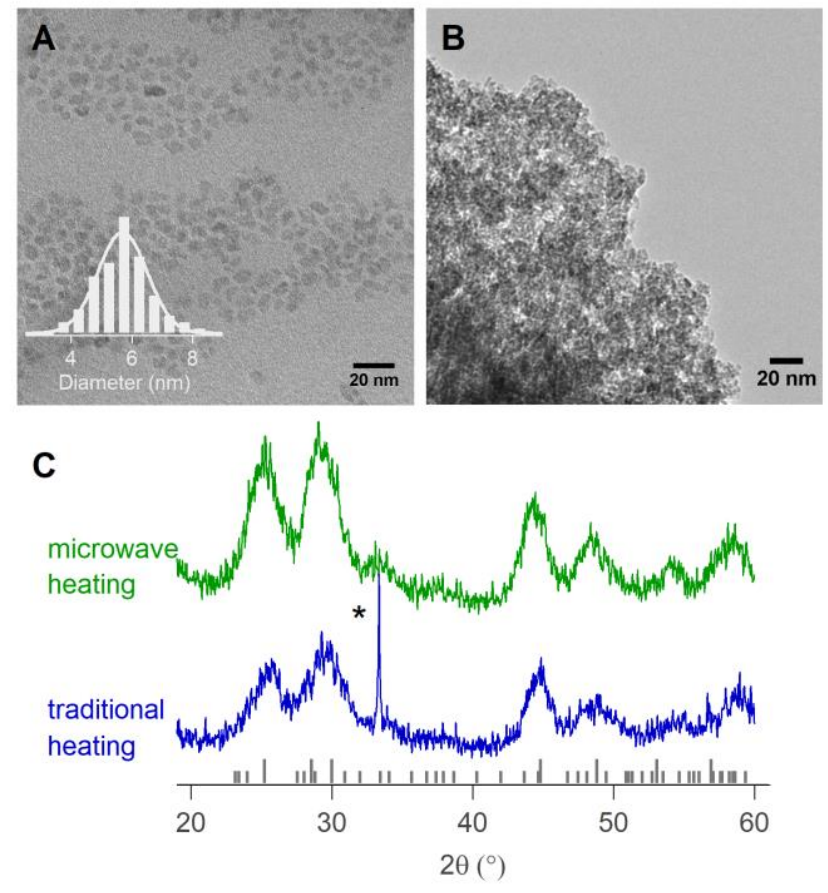

Figure 1: A) TEM image of non-agglomerated $\mathrm{TiO}_{2}-\mathrm{B}$ NCs synthesized in the microwave at $160{ }^{\circ} \mathrm{C}$ for 30 min, stabilized with OLAm/OLAc, after 2 purification steps. B) $\mathrm{TiO}_{2}-\mathrm{B}$ NCs synthesized in a traditional oven at $160{ }^{\circ} \mathrm{C}$ for $30 \mathrm{~min}$. C) XRD pattern of microwave synthesized $\left(160{ }^{\circ} \mathrm{C} / 30 \mathrm{~min}\right) \mathrm{TiO}_{2}-\mathrm{B}$ NCs. D) XRD pattern of $\mathrm{TiO}_{2}-\mathrm{B} \mathrm{NCs}$ in the autoclave at $160^{\circ} \mathrm{C}$ for $30 \mathrm{~min}$ In both $\mathrm{C}$ and $\mathrm{D}$ only diffraction patterns of the Bronze phase are identified, with reference pattern ICSD \# 657748. The sharp peak * at a $2 \theta$ of $33^{\circ}$ can be assigned to the $\mathrm{Si}$-substrate used for the XRD acquisition.

Controlling nanocrystal size and aggregation. Considering the success of microwave heating in obtaining colloidally stable $\mathrm{TiO}_{2}-\mathrm{B} \mathrm{NCs}$, we sought a systematic method to understand the factors that (1) determine agglomeration and (2) tune the size of the NCs. Therefore, we turned to a design of experiments, Definitive Screening Design (DSD), as this is a powerful method to determine the descriptive equation of each material property (called the response, Y) with a minimal amount of experiments. ${ }^{47} \mathrm{We}$ choose to monitor two responses; the $\mathrm{NC}$ size, quantified by its diameter as determined from TEM ( $\left.\mathrm{Y}_{1}\right)$ and the agglomeration of the NCs after surface modification with oleic acid and oleylamine, quantified by the Z-average parameter from Dynamic Light Scattering $\left(\mathrm{Y}_{2}\right)$. Four factors were identified as potentially 
significant in affecting these responses: the amount of glycolic acid $\left(\chi_{1}\right)$, the amount of sulfuric acid $\left(\chi_{2}\right)$, the reaction temperature $\left(\chi_{3}\right)$ and the reaction time $\left(\chi_{4}\right)$. The concentration of Ti metal was kept constant at $0.25 \mathrm{~mol} \mathrm{~L}^{-1}$.

DSD is a three-level factor design and -1, 0 and 1 factor levels are chosen based on preliminary experiments (see Table 1 and SI). The limitations were set based on the formation of alien phases, for example at $200^{\circ} \mathrm{C}$, anatase starts to form. Therefore, the maximum temperature $(+1$, $\left.\chi_{3}\right)$ was set at $180{ }^{\circ} \mathrm{C}$. For the present four factor $(\mathrm{k}=4)$ analysis, 13 DSD experiments $(3 \mathrm{k}+1)$ are performed. ${ }^{46}$ The different experiments and responses are shown in Table 1 and the corresponding TEM and DLS experiments can be found in the SI.

Table 1. Definitive screening design of 4 factors: amount of glycolic acid, $\chi_{1}$, amount of sulfuric acid, $\chi_{2}$, the reaction temperature, $\chi_{3}$ and the reaction time, $\chi_{4}$. The obtained results are the nanocrystals size (diameter), $Y_{1}$ and the agglomeration quantified by the Z-average value from DLS experiments, $\mathrm{Y}_{2}$. Both the training set entries (T1-T13) as the control experiments (C1-C2) are listed.

\begin{tabular}{|c|c|c|c|c|c|c|}
\hline Entry & $\begin{array}{c}\chi_{1} \\
\text { Glycolic acid } \\
(\mathrm{mmol})\end{array}$ & $\begin{array}{c}\chi_{2} \\
\mathrm{H}_{2} \mathrm{SO}_{4} \\
\text { (mmol) }\end{array}$ & $\begin{array}{c}\chi_{3} \\
\mathbf{T} \\
\left({ }^{\circ} \mathbf{C}\right)\end{array}$ & $\begin{array}{c}\chi_{4} \\
\text { Time } \\
(\min )\end{array}$ & $\begin{array}{c}Y_{1} \\
\text { Size } \\
(\mathbf{n m})\end{array}$ & $\begin{array}{l}\mathrm{Y}_{2} \\
\mathrm{Z}-\mathbf{a v} . \\
\text { (nm) }\end{array}$ \\
\hline $\mathrm{T} 1$ & 1.5 & 2.8 & 180 & 45 & $7.8 \pm 1.5$ & 209 \\
\hline $\mathrm{T} 2$ & 1.5 & 1.6 & 130 & 5 & $5.0 \pm 1.1$ & 74.15 \\
\hline $\mathrm{T} 3$ & 2 & 2.2 & 130 & 45 & $5.5 \pm 1.6$ & 24.24 \\
\hline $\mathrm{T} 4$ & 1 & 2.2 & 180 & 5 & $6.2 \pm 1.4$ & 20.43 \\
\hline T5 & 2 & 1.6 & 155 & 5 & $6.5 \pm 1.5$ & 27.78 \\
\hline T6 & 1 & 2.8 & 155 & 45 & $6.4 \pm 1.3$ & 221.6 \\
\hline $\mathrm{T} 7$ & 2 & 2.8 & 130 & 25 & $5.5 \pm 2.3$ & 132.6 \\
\hline T8 & 1 & 1.6 & 180 & 25 & $5.8 \pm 1.4$ & 33.15 \\
\hline T9 & 2 & 2.8 & 180 & 5 & $7.0 \pm 1.9$ & 121.8 \\
\hline $\mathrm{T} 10$ & 1 & 1.6 & 130 & 45 & $4.5 \pm 1.3$ & 18.85 \\
\hline $\mathrm{T} 11$ & 2 & 1.6 & 180 & 45 & $5.1 \pm 1.6$ & 99.82 \\
\hline $\mathrm{T} 12$ & 1 & 2.8 & 130 & 5 & $3.6 \pm 1.2$ & 19.67 \\
\hline $\mathrm{T} 13$ & 1.5 & 2.2 & 155 & 25 & $5.4 \pm 1.9$ & 55.49 \\
\hline $\mathrm{C} 1$ & 1 & 2.8 & 130 & 5 & $2.8 \pm 0.5$ & 16.14 \\
\hline $\mathrm{C} 2$ & 1.5 & 2.2 & 190 & 5 & $8.0 \pm 1.0$ & 17.61 \\
\hline
\end{tabular}

The data was analyzed by the statistical software package JMP ${ }^{\circledR}$ and in table S5 the significant effects (main, interaction and quadratic) are listed. The corresponding scaled model equations for the $\mathrm{NC}$ size $\left(\mathrm{Y}_{1}\right.$ in $\left.\mathrm{nm}\right)$ and agglomeration $\left(\mathrm{Y}_{2}\right.$ in $\left.\mathrm{nm}\right)$ are respectively:

$$
\begin{aligned}
Y_{1}= & 5.716+0.306\left(\frac{\chi_{1}-1.5}{0.5}\right)+0.47\left(\frac{\chi_{2}-2.2}{0.6}\right)+0.77\left(\frac{\chi_{3}-155}{25}\right)-0.0307\left(\frac{\chi_{1}-1.5}{0.5}\right)\left(\frac{\chi_{3}-155}{25}\right) \\
& +0.6743\left(\frac{\chi_{2}-2.2}{0.6}\right)\left(\frac{\chi_{3}-155}{25}\right)
\end{aligned}
$$




$$
\begin{aligned}
Y_{2}= & 33.386+45.092\left(\frac{\chi_{2}-2.2}{0.6}\right)+30.968\left(\frac{\chi_{4}-25}{20}\right)+62.455\left(\frac{\chi_{2}-2.2}{0.6}\right)\left(\frac{\chi_{2}-2.2}{0.6}\right) \\
& -34.049\left(\frac{\chi_{2}-2.2}{0.6}\right)\left(\frac{\chi_{4}-25}{20}\right)
\end{aligned}
$$

As apparent from the coefficients of equation 1, the NC size is about equally affected by the glycolic acid concentration and the sulfuric acid concentration. However, the main factor determining the $\mathrm{NC}$ size is the reaction temperature $\chi_{3}$. The higher the temperature, the larger the NCs, within the $130-180{ }^{\circ} \mathrm{C}$ range. The reaction time $\chi_{4}$ is even absent from equation 1 , indicating a very fast nanocrystal nucleation and growth. This is confirmed by the almost quantitative yield of the reaction, even for the shortest reaction time studied (5 minutes, yield: $95 \%)$.

The extent of agglomeration is determined by the reaction time $\chi_{4}$ and the sulfuric acid concentration $\chi_{2}$ (Eq. 2). Given the slow heating and cooling rate of a stainless-steel bomb, this explains the extensive agglomeration of NCs synthesized in traditional oven. Indeed, the effective reaction time at elevated temperature is prolonged by these slow rates and consequently, the aggregated nanocrystals are useless for their envisioned applications. ${ }^{14,27,28}$ Alternatively, the microwave achieves a heating rate of $100{ }^{\circ} \mathrm{C} / \mathrm{min}$ and cooling rate of 50 ${ }^{\circ} \mathrm{C} / \mathrm{min}$. We conclude that microwave heating is superior in synthesizing aggregate-free $\mathrm{TiO}_{2}-$ B NCs as it allows for very short reaction times.

In Figure 2A the size predicted by the model is plotted against the experimental size. An excellent correlation for all measurements is found, indicating the good fit of the response equation (3). The dispersion of the predicted vs. experimental agglomeration is a bit higher (Figure 2B), possibly due to external factors affecting the DLS measurements. Nevertheless a clear influence of the reaction time $\chi_{4}$ and sulfuric acid concentration $\chi_{2}$ can be expected. For clarity, we define non-agglomerated NCs as samples with a Z-average smaller than $50 \mathrm{~nm}$ (grey area in Figure 2B). 

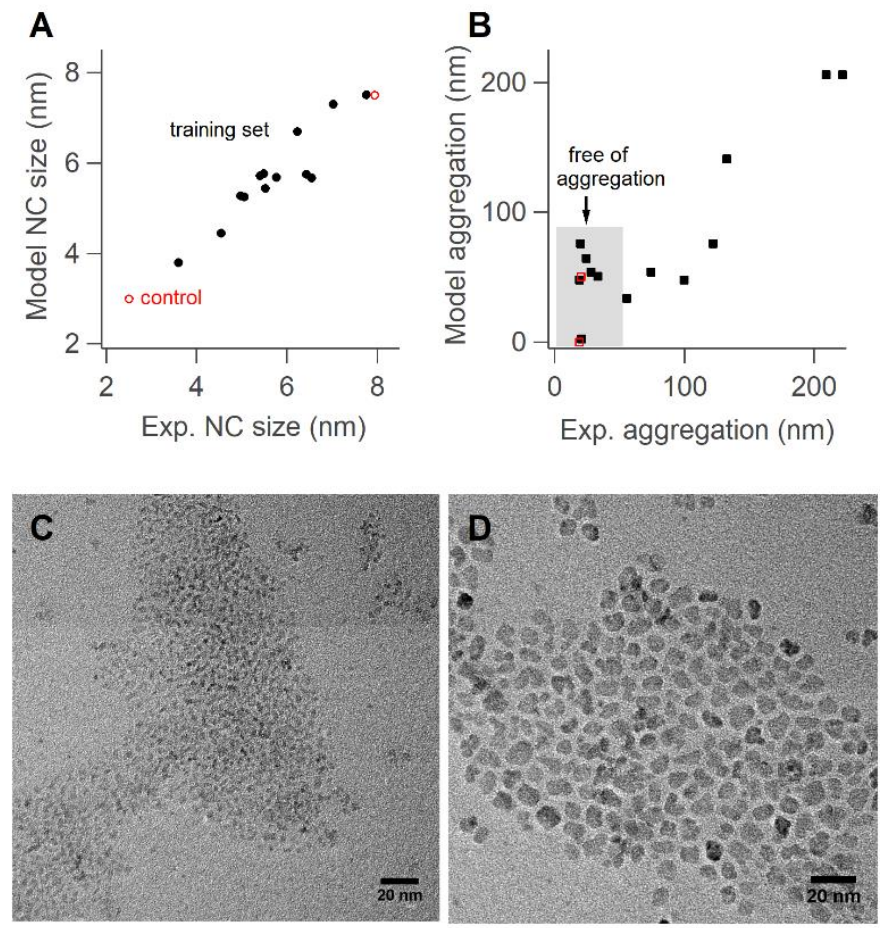

Figure 2. (A) Predicted vs. experimental NC size and (B) the predicted agglomeration vs. experimental agglomeration. (C) TEM image of $\mathrm{TiO}_{2}-\mathrm{B}$ NCs synthesized via the parameters of $\mathrm{C} 1$ (Table2), and (D) NCs synthesized with parameters C2.

Having established our model using a training set of experiments, we turned to validating the model with control experiments (C1-C2, Table 1). We aimed a synthesizing two NCs sizes (3 and $7.5 \mathrm{~nm}$ ) without aggregation. Based on the previously obtained insights, we chose a reaction time of 5 minutes to avoid irreversible agglomeration. In principle, the sulfuric acid concentration $\chi_{2}$ has a quadratic effect on $\mathrm{Y}_{2}$, and thus the lowest agglomeration is found around the ' 0 ' factor level, corresponding to $2.2 \mathrm{mmol}$. However, to obtain $3 \mathrm{~nm} \mathrm{NCs}$, the dependency of $Y_{1}$ to $\chi_{2}$, obliged us to fix the amount of sulfuric acid at $2.8 \mathrm{mmol}$. The temperature was set at $130{ }^{\circ} \mathrm{C}$ for $3 \mathrm{~nm} \mathrm{NCs}$ and at $190{ }^{\circ} \mathrm{C}$ for $7.5 \mathrm{~nm}$ NCs. Note that we venture outside the DSD training set window $\left(130-180^{\circ} \mathrm{C}\right)$ to maximize the nanocrystal size. This is warranted because very short reaction times were used, thus precluding anatase formation. In Figure $2 \mathrm{C}$ and $2 \mathrm{D}$, TEM images of the resulting NCs of experiment $\mathrm{C} 1$ and $\mathrm{C} 2$ are displayed. Both controls only produced NCs with the Bronze phase (Figure S3-S4), combined with very low Z-average values (Table 1). Also from Figure 2A and 2B, the success of these control experiments is clear since they fit the trend of the training set. We conclude that this model is a powerful tool to rationally design the synthesis of aggregate-free $\mathrm{TiO}_{2}$-bronze $\mathrm{NCs}$ of any desired size between 2.8 and 8 nm.

Elucidating the surface modification. To study the $\mathrm{TiO}_{2}-\mathrm{B} \mathrm{NC}$ surface chemistry in detail, $5.5 \pm 1.9 \mathrm{~nm}$ NCs are synthesized (see experimental). The $\mathrm{TiO}_{2}-\mathrm{B} \mathrm{NC}$ precipitate is isolated 
and cannot be directly dispersed in any solvent. However, when triethylamine is added, the NCs are readily stabilized in the base and can be subsequently dispersed in either water, methanol, ethanol, chloroform or dimethyl formamide. In the ${ }^{1} \mathrm{H}$ spectrum of such a $\mathrm{TiO}_{2}-\mathrm{B} \mathrm{NC}$ dispersion in $\mathrm{D}_{2} \mathrm{O}$ (Figure $3 \mathrm{~A}$ ), the resonances of protonated triethylamine are easily recognized at 1 and $2.7 \mathrm{ppm}$. The singlet at $3.8 \mathrm{ppm}$ was attributed to desorbed glycolic acid and this assignment is corroborated by 2D HSQC and HMBC analysis (Figure S10 and S11). Any adsorbed glycolic acid would be undetectable in NMR as the reduced mobility at the surface enhances the rate of $\mathrm{T}_{2}$ relaxation. ${ }^{51}$ We thus infer that the $\mathrm{NC}$ were originally capped by glycolic acid and in water, hydroxide ions have at least partly displaced glycolic acid. The ligand exchange is facilitated by the high concentration of hydroxide and glycolic acid's high solubility in water. In comparison, no glycolic acid is released in chloroform from the surface of triethylamine stabilized NCs (Figure S12). The triethylamine stabilized NCs feature a negative zeta potential $(-35 \mathrm{mV})$ in water, indicating an electrostatic stabilization mechanism. Upon decreasing the $\mathrm{pH}$, the NCs destabilized gradually and at a pH of 3.85 the isoelectric point is reached (Figure 3B), which is in compliance with the slightly acidic nature of the surface bound $\mathrm{OH}$ 's of $\mathrm{TiO}_{2}{ }^{52,53}$ At lower $\mathrm{pH}$, the zeta potential increases again in absolute value, now with a positive value. We conclude that the role of triethylamine in the stabilization is simply to provide a basic environment. To confirm this hypothesis, the surface modification and transfer to water was successfully performed with 1,8-diazabicyclo[5.4.0]undec-7-ene (DBU). DBU is a sterically hindered base, only capable of deprotonation and cannot directly coordinate to the surface. 

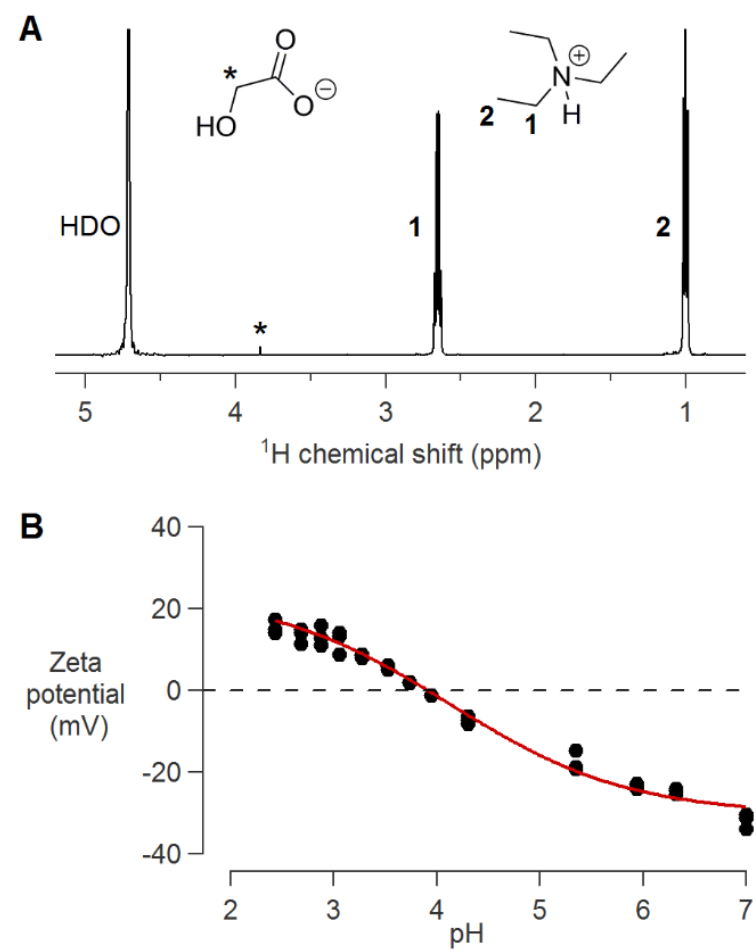

Figure 3. (A) ${ }^{1} \mathrm{H}$ NMR spectrum of $\mathrm{TiO}_{2}-\mathrm{B}$ NCs stabilized with triethylamine in $\mathrm{D}_{2} \mathrm{O}$. (B) Zeta-potential titration of $\mathrm{TiO}_{2}-\mathrm{B}$ NCs stabilized with DBU in $\mathrm{H}_{2} \mathrm{O}(\mathrm{pH} 7.1)$, showing a Zeta-potential of $-35 \mathrm{mV}$. By adding a diluted $\mathrm{HCl}$ solution, the NCs gradually precipitate.

When oleylamine is employed as a base, the NCs can also be dispersed in chloroform. Unfortunately, as indicated by DLS (Figure 4), the solvodynamic diameter (27 $\mathrm{nm}$ ) is much larger than the primary particle size $(5.5 \mathrm{~nm})$, indicating some residual degree of aggregation. However, the addition of oleic acid causes a significant decrease in the solvodynamic diameter to about $10 \mathrm{~nm}$. This is the expected value since the inorganic core $(5.5 \mathrm{~nm})$ plus twice the ligand shell $(2 \mathrm{~nm})$ is about $9.5 \mathrm{~nm}$. Interestingly, when only oleic acid is used, without oleylamine, the NCs are not stable and precipitate.

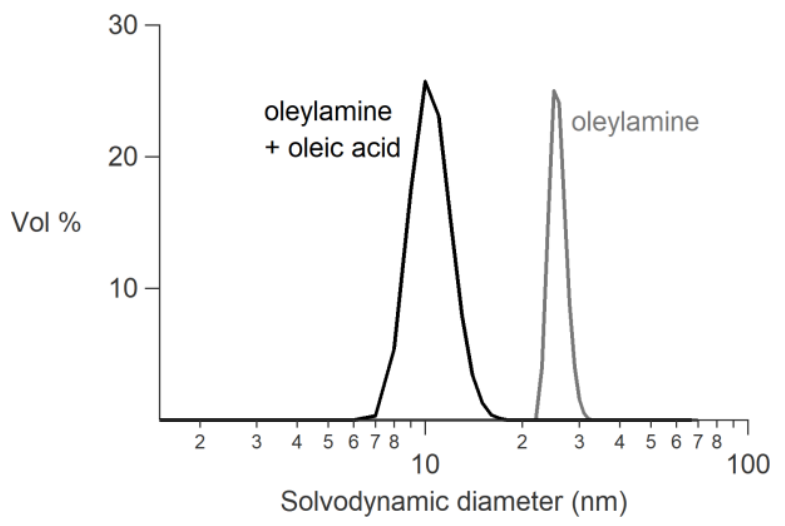

Figure 4. Size distribution by DLS of $\mathrm{TiO}_{2}-\mathrm{B}$ NCs in chloroform, when either only oleylamine is added or when both oleylamine and oleic acid are added 
To further elucidate the roles of the different surfactants, we turned again to solution ${ }^{1} \mathrm{H}$ NMR. It is difficult to distinguish oleylamine from oleic acid in NMR since both have the same characteristic alkene resonance. Therefore, for the purpose of the NMR experiments, either the combination of oleylamine with dodecanoic acid or the combination of octylamine with oleic acid was used for the surface modification. ${ }^{54}$ After functionalization, the NCs were purified 4 times using acetone to remove unbound ligands. In both cases, the ${ }^{1} \mathrm{H}$ NMR spectrum (Figure $5 \mathrm{~A}$ and $5 \mathrm{~B}$ ) features an broadened alkene resonance, indicating that both the amine and the carboxylate remain bound to the nanocrystal surface. Therefore, we hypothesized that the NC surface is stabilized by ion pairs of alkylammonium carboxylate, a binding motif already observed in $\mathrm{CdSe}^{55,56}$ and $\mathrm{CsPbr}_{3}{ }^{57}$ nanocrystals. Pulsed Field Gradient spectroscopy further confirmed the binding of the ion pair to the NC surface. A diffusion coefficient of $115 \pm 4 \mu \mathrm{m}^{2} / \mathrm{s}$ is obtained for both oleic acid and oleylamine (Figure S14 and S15). This corresponds to a solvodynamic diameter, $\mathrm{d}_{\mathrm{s}}$, of $7.0 \pm 0.3 \mathrm{~nm}$ and is slightly smaller than what would be expected of a $5.5 \mathrm{~nm}$ crystal with oleate ligands $\left(\mathrm{d}_{\mathrm{s}}=9.5 \mathrm{~nm}\right)$. This indicates some degree of exchange between a bound and free state with however the highest contribution from the bound state. Due to the small contribution of the free state, the resonances close to the surface are detected albeit excessively broadened. Around $8 \mathrm{ppm}$, the characteristic ammonium resonance is observed, further confirming the presence of an alkylammonium carboxylate ion pair. The relatively tight binding of the ion pair stands in contrast to the high lability of the ligand shell of $\mathrm{CsPbBr}_{3}$ nanocrystals, ${ }^{57}$ and the $\mathrm{TiO}_{2}$ - $\mathrm{B}$ dispersion features an excellent long term colloidal stability. Note that the dispersion needs to be kept away from light due to the high photocatalytic activity of the $\mathrm{TiO}_{2}$-B NCs. Indeed, after exposure of the NCs to natural sunlight for 8 hours, the NMR spectrum clearly features degradation products of the organic ligands (Figure S16). 

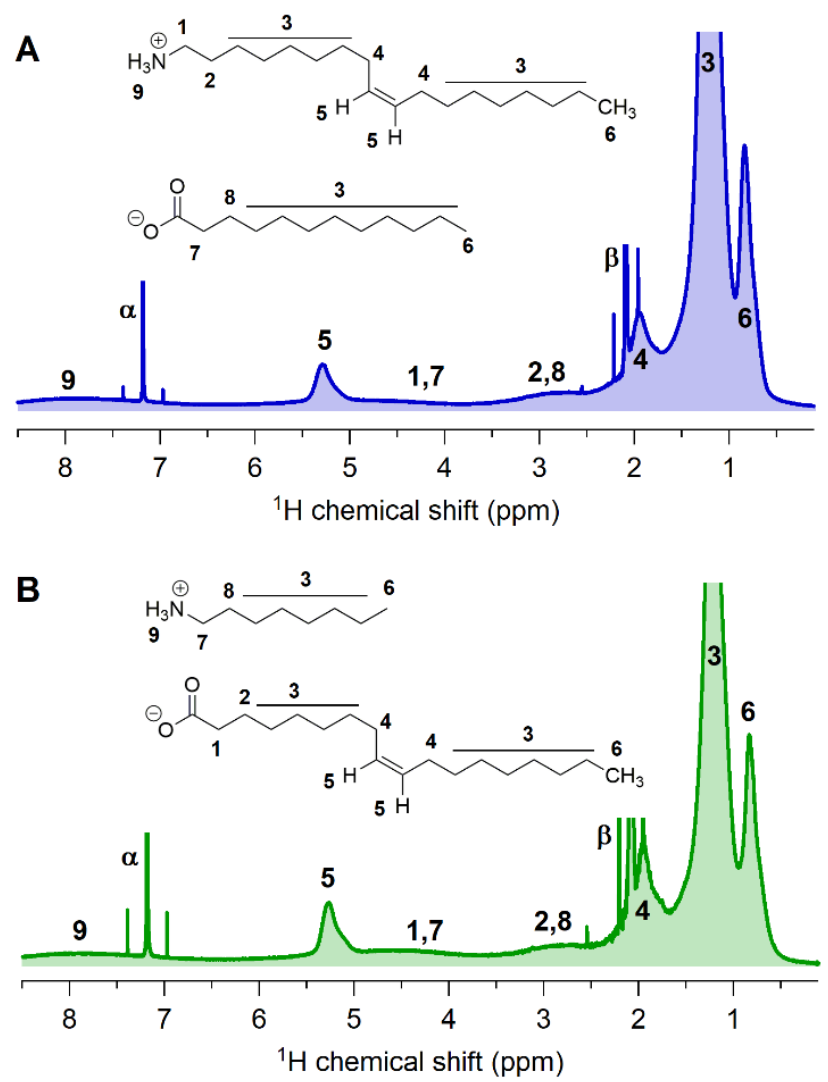

Figure 5. ${ }^{1} \mathrm{H}$ NMR spectrum of $\mathrm{TiO}_{2}-\mathrm{B} \mathrm{NCs}$ in $\mathrm{CDCl}_{3}$ stabilized with (A) dodecanoic acid and oleylamine after 3 purification steps and (B) octylamine and oleic acid after 3 purification steps. The alpha resonance refers to chloroform while the beta resonance corresponds to acetone.

To further confirm the stoichiometry of the ion pair, we sought to remove the ligands from the nanocrystals surface. Surprisingly, established procedures such as stripping with trifluoroacetic acid ${ }^{58} \mathrm{NOBF}_{4},{ }^{59}$ or Meerwein's salt ${ }^{60}$ did not succeed in separating the ligands from the NCs. Only treatment of the $\mathrm{NCs}$ with $\mathrm{BF}_{3}$ according to Doris et al. ${ }^{48}$ resulted in precipitation of the nanocrystals. The latter could be redispersed in polar solvents such as DMF with a cationic surface charge (zeta potential $=+52 \mathrm{mV}$ ). The supernatant with the stripped ligand was evaporated and measured in NMR (Figure S17). From the relative integration of the amine and acids signals we conclude a 1:1 ratio of amine to carboxylic acid.

\section{Discussion of the surface model}

To rationalize the conclusions from the Design of Experiment and the observations during the surface functionalization experiments, we strived to obtain a complete surface chemistry picture. In this respect, the surface of the as-synthesized NCs is difficult to characterize because the candidate surface species are either NMR inactive $\left(\mathrm{SO}_{4}{ }^{2-}\right)$ or too small and close to the surface to be observed $\left(\mathrm{H}^{+}\right.$, glycolic acid). ${ }^{51}$ However, CHNS analysis clear showed the presence of sulfur in the as-synthesized nanocrystals (Figure S18), strongly suggesting surface 
adsorbed sulfate ions. In addition, glycolic acid is clearly released from the surface upon stabilization of the NCs in water. Therefore, both species are present on the NC surface and it is thus no surprise that their concentration during synthesis plays a role in the size and degree of aggregation (vide supra). Finally, a brønsted base such as oleylamine is able to partially stabilize the NCs in chloroform and an ammonium resonance is observed in the ${ }^{1} \mathrm{H}$ NMR spectrum, proving the presence of surface protons. Combined, these conclusions give rise to a surface chemistry picture with surface adsorbed protons, sulfate and glycolate moieties (Scheme 2).

A

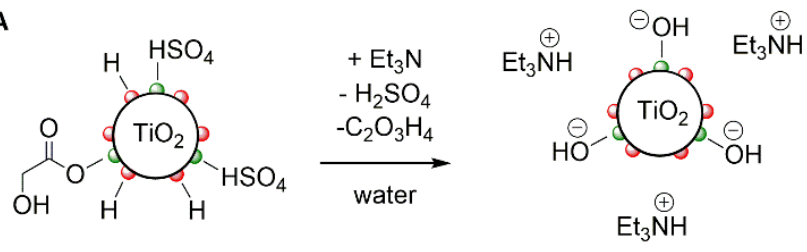

B
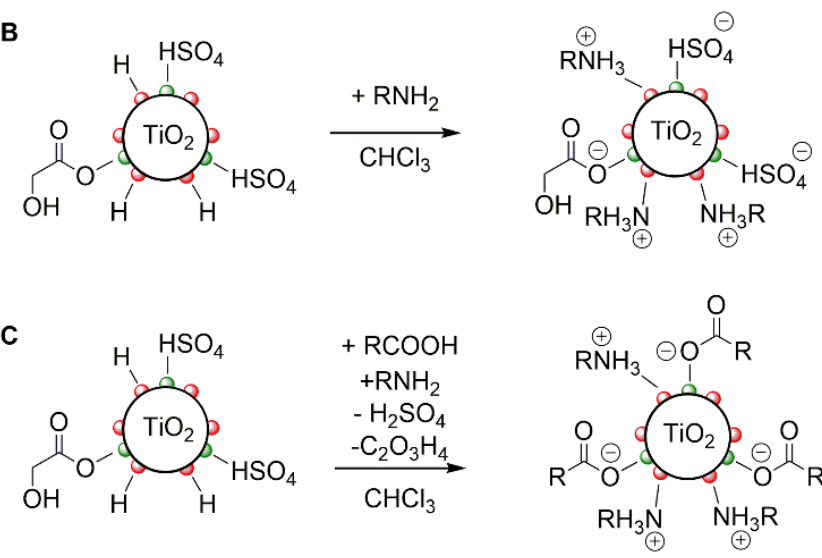

- titanium o oxygen

Scheme 2. (A) Surface functionalization in water with triethylamine. Given the high solubility and acidity of sulfuric acid in water, we hypothesize desorption from the surface upon addition of base. (B) Surface functionalization in chloroform with oleylamine. (C) Surface functionalization in chloroform with oleylamine and oleic acid. These cartoons are simply intended to represent surface species and surface reactivity and are not an accurate representation of the true binding mode, which is probably chelating for oleate or involves a five-membered ring for glycolate.

In water, the $\mathrm{TiO}_{2}-\mathrm{B}$ NCs are charge stabilized with hydroxide ions due to the basic environment created by triethylamine (Scheme 2A). The glycolic acid and sulfuric acid on the surface of the as-synthesized NCs desorb due to their high solubility in water. In contrast, functionalization with oleylamine in chloroform does not lead to desorption of these surface species. Rather, oleylamine is protonated and remains associated with the surface (Scheme 2B). When both oleylamine and oleic acid are added in excess, CHNS analysis (Figure S19) shows that after purification the NCs don't contain sulfur anymore, suggesting an exchange of sulfate for oleate ligands (Scheme 2C). Glycolic acid is detected in the supernatant of the washed cycles 
(Figure S20) and we conclude that it is also replaced by oleate ligands. At first sight, the replacement of inorganic sulfate ions for carboxylate ligands is very similar to the surface modification of hydrogen chloride capped hafnium and zirconium oxide nanocrystals. ${ }^{39,40,50}$ However, in those cases, the final surface chemistry consisted of dissociated carboxylic acids. ${ }^{39}$ In addition, a very large excess of alkylamine is necessary to form an ion pair and remove the carboxylate from the surface. ${ }^{61}$ In contrast, here we consistently retrieve tightly bound ion pairs of alkylammonium carboxylates on the NCs, even if a threefold excess of carboxylic acid with respect to amine is used. This indicates a higher acidity of the titania surface, which is corroborated by its lower isoelectric point (3.85 for $\mathrm{TiO}_{2}$ - $\mathrm{B}$ and 8.3 for $\mathrm{HfO}_{2}$ ) ${ }^{62}$ Importantly, since the isoelectric point does not change significantly among the titania polymorphs ${ }^{63}$, these results suggest that dissociated carboxylic acids on titania nanocrystals are impossible to obtain in the presence of amines. NCs that were previously assigned as such are more likely stabilized by an alkylammonium carboxylate ion pair. ${ }^{64}$ Our results also hint at the almost molecular $\mathrm{acid} / \mathrm{b}$ ase behavior of metal oxide nanocrystals where the isoelectric point is equivalent to a pKa. From this point of view, the dissociative behavior of acids on titania and hafnia can be easily rationalized. Strong acids such hydrochloric acid or sulfuric acid have negative pKa's, clearly lower than the "pKa" of titania (3.85) or hafnia (8.3) and consequently dissociate on either surface. However, carboxylic acids $(\mathrm{pKa}=4.75)$ are one order of magnitude less acidic than titania while still several orders more acidic than hafnia. Therefore, carboxylic acids don't dissociate on titania while they do on hafnia. With these fundamental insights, we can precisely predict the surface chemistry of any metal oxide - provided the isoelectric point is known or measured - and turn them in valuable building blocks.

\section{Conclusion}

For the first time, we present a size tuning strategy towards colloidally stable $\mathrm{TiO}_{2}$-bronze nanocrystals. By performing a design of experiments, we showed that the main parameter influencing the NC size is the reaction temperature while agglomeration is determined by the reaction time and the sulfuric acid concentration. Therefore, by using fast microwave heating, irreversible aggregation could be completely avoided and several subsequent surface functionalizations delivered colloidally stable NCs in either polar solvents with a positive or negative surface charge or in nonpolar solvents with steric surfactants. With a microwave temperature ranging from $130{ }^{\circ} \mathrm{C}-180{ }^{\circ} \mathrm{C}, \mathrm{NCs}$ in the size range of $3-8 \mathrm{~nm}$ can be selectively synthesized. As-synthesized NCs feature surface adsorbed glycolic acid and sulfuric acid, which are replaced by an oleate ligand during the surface modification in a nonpolar solvent. 
Surprisingly, the NCs are not stabilized by dissociated carboxylic acid like $\mathrm{HfO}_{2}$ and $\mathrm{ZrO}_{2} \mathrm{NCs}$ but rather by alkylammonium carboxylate ion pairs. We have correlated this unique feature to the higher acidity of titania and therefore, it also applies to the other polymorphs of titania. As such, these results will not only spur research into the application of $\mathrm{TiO}_{2}$-bronze $\mathrm{NCs}$ in batteries or photocatalysis but also provide guidance in the functionalization of metal oxide surfaces in general.

\section{Aknowledgements}

Ghent University is thanked for funding the research performed for this paper. We thank F. Deruyck for discussing the definitive screening design. Jonathan Watté and Tom Planckaert are thanked for their help with XRD and CHNS analysis. Jonathan De Roo acknowledges support from the Belgian American Education Foundation (BAEF), Fulbright, Ghent University and the European Union's Framework Program for Research and Innovation Horizon 2020 (20142020) under the Marie Skłodowska-Curie Grant Agreement COMPASS No. 691185.

\section{References}

1. Oregan, B.; Gratzel, M., A LOW-COST, HIGH-EFFICIENCY SOLAR-CELL BASED ON DYESENSITIZED COLLOIDAL TIO2 FILMS. Nature 1991, 353, 737-740.

2. De Trizio, L.; Buonsanti, R.; Schimpf, A. M.; Llordes, A.; Gamelin, D. R.; Simonutti, R.; Milliron, D. J., Nb-Doped Colloidal TiO2 Nanocrystals with Tunable Infrared Absorption. Chem. Mat. 2013, 25, 3383-3390.

3. Chou, T. P.; Zhang, Q. F.; Russo, B.; Cao, G. Z., Enhanced light-conversion efficiency of titanium-dioxide dye-sensitized solar cells with the addition of indium-tin-oxide and fluorinetin-oxide nanoparticles in electrode films. J. Nanophotonics 2008, 2(1), 23511-23522.

4. Paulose, M.; Varghese, O. K.; Mor, G. K.; Grimes, C. A.; Ong, K. G., Unprecedented ultrahigh hydrogen gas sensitivity in undoped titania nanotubes. Nanotechnology 2006, 17, 398402.

5. Hossain, M. K.; Koirala, A. R.; Akhtar, U. S.; Song, M. K.; Yoon, K. B., First Synthesis of Highly Crystalline, Hexagonally Ordered, Uniformly Mesoporous TiO2-B and Its Optical and Photocatalytic Properties. Chem. Mat. 2015, 27, 6550-6557.

6. Zhang, Y.; Xing, Z. P.; Liu, X. F.; Li, Z. Z.; Wu, X. Y.; Jiang, J. J.; Li, M.; Zhu, Q.; Zhou, W., Ti3+ Self-Doped Blue TiO2(B) Single-Crystalline Nanorods for Efficient Solar-Driven Photocatalytic Performance. ACS Appl. Mater. Interfaces 2016, 8, 26851-26859.

7. Xiang, G. L.; Li, T. Y.; Zhuang, J.; Wang, X., Large-scale synthesis of metastable TiO2(B) nanosheets with atomic thickness and their photocatalytic properties. Chem. Commun. 2010, 46, 6801-6803.

8. Giannuzzi, R.; Manca, M.; De Marco, L.; Belviso, M. R.; Cannavale, A.; Sibillano, T.; Giannini, C.; Cozzoli, P. D.; Gigli, G., Ultrathin TiO2(B) Nanorods with Superior Lithium-lon Storage Performance. ACS Appl. Mater. Interfaces 2014, 6, 1933-1943. 
9. Etacheri, V.; Yourey, J. E.; Bartlett, B. M., Chemically Bonded TiO2-Bronze Nanosheet/Reduced Graphene Oxide Hybrid for High-Power Lithium Ion Batteries. ACS Nano 2014, 8, 1491-1499.

10. Goriparti, S.; Miele, E.; De Angelis, F.; Di Fabrizio, E.; Zaccaria, R. P.; Capiglia, C., Review on recent progress of nanostructured anode materials for Li-ion batteries. J. Power Sources 2014, 257, 421-443.

11. Liu, Z.; Andreev, Y. G.; Armstrong, A. R.; Brutti, S.; Ren, Y.; Bruce, P. G., Nanostructured TiO2(B): the effect of size and shape on anode properties for Li-ion batteries. Prog. Nat. Sci. 2013, 23, 235-244.

12. Yan, W. W.; Zou, Y.; Zhou, H.; Wang, L. L.; Meng, X. F., Synergistic effect of sodium ions and fluoride ions on synthesis of pure-phase TiO2(B) nanorings. J. Nanopart. Res. 2017, 19, 110.

13. Ren, G. F.; Hoque, M. N. F.; Liu, J. W.; Warzywoda, J.; Fan, Z. Y., Perpendicular edge oriented graphene foam supporting orthogonal TiO2(B) nanosheets as freestanding electrode for lithium ion battery. Nano Energy 2016, 21, 162-171.

14. Dylla, A. G.; Henkelman, G.; Stevenson, K. J., Lithium Insertion in Nanostructured TiO2(B) Architectures. Accounts Chem. Res. 2013, 46, 1104-1112.

15. Dawson, J. A.; Robertson, J., Improved Calculation of Li and $\mathrm{Na}$ Intercalation Properties in Anatase, Rutile, and TiO2(B). J. Phys. Chem. C 2016, 120, 22910-22917.

16. Wilkening, M.; Lyness, C.; Armstrong, A. R.; Bruce, P. G., Diffusion in Confined Dimensions: Li+ Transport in Mixed Conducting TiO2-B Nanowires. J. Phys. Chem. C 2009, 113, 4741-4744.

17. Bruce, P. G.; Scrosati, B.; Tarascon, J. M., Nanomaterials for rechargeable lithium batteries. Angew. Chem.-Int. Edit. 2008, 47, 2930-2946.

18. Marchand, R.; Brohan, L.; Tournoux, M., TIO2(B) A NEW FORM OF TITANIUM-DIOXIDE AND THE POTASSIUM OCTATITANATE K2TI8O17. Mater. Res. Bull. 1980, 15, 1129-1133.

19. Armstrong, A. R.; Armstrong, G.; Canales, J.; Bruce, P. G., TiO2-B nanowires. Angew. Chem.-Int. Edit. 2004, 43, 2286-2288.

20. Li, K. K.; Li, B. H.; Wu, J. X.; Kang, F. Y.; Kim, J. K.; Zhang, T. Y., Ultrafast-Charging and Long-Life Li-Ion Battery Anodes of Tio(2)-B and Anatase Dual-Phase Nanowires. ACS Appl. Mater. Interfaces 2017, 9, 35917-35926.

21. Armstrong, G.; Armstrong, A. R.; Canales, J.; Bruce, P. G., Nanotubes with the TiO2-B structure. Chem. Commun. 2005, 2454-2456.

22. Chen, C. J.; Hu, X. L.; Hu, P.; Qiao, Y.; Qie, L.; Huang, Y. H., lonic-Liquid-Assisted Synthesis of Self-Assembled TiO2-B Nanosheets under Microwave Irradiation and Their Enhanced Lithium Storage Properties. Eur. J. Inorg. Chem. 2013, 2013, 5320-5328.

23. Wang, J. F.; Xie, J. J.; Jiang, Y. M.; Zhang, J. J.; Wang, Y. G.; Zhou, Z. F., Preparation of mesoporous TiO2-B nanowires from titanium glycolate and their application as an anode material for lithium-ion batteries. J. Mater. Sci. 2015, 50, 6321-6328.

24. Zhen, M. M.; Guo, S. Q.; Gao, G. D.; Zhou, Z.; Liu, L., TiO2-B nanorods on reduced graphene oxide as anode materials for $\mathrm{Li}$ ion batteries. Chem. Commun. 2015, 51, 507-510.

25. Wu, L. M.; Bresser, D.; Buchholz, D.; Passerini, S., Nanocrystalline TiO2(B) as Anode Material for Sodium-Ion Batteries. J. Electrochem. Soc. 2015, 162, A3052-A3058.

26. Prochazka, J.; Kavan, L.; Zukalova, M.; Frank, O.; Kalbac, M.; Zukal, A.; Klementova, M.; Carbone, D.; Graetzel, M., Novel Synthesis of the TiO2(B) Multilayer Templated Films. Chem. Mat. 2009, 21, 1457-1464. 
27. Kobayashi, M.; Petrykin, V. V.; Kakihana, M., One-step synthesis of TiO2(B) nanoparticles from a 'Water-Soluble titanium complex. Chem. Mat. 2007, 19, 5373-5376.

28. Shang, Y. H.; Lin, X. J.; Lu, X.; Huang, T.; Yu, A. H., Nano-TiO2(B) coated LiMn2O4 as cathode materials for lithium-ion batteries at elevated temperatures. Electrochim. Acta 2015, 156, 121-126.

29. Dylla, A. G.; Lee, J. A.; Stevenson, K. J., Influence of Mesoporosity on Lithium-Ion Storage Capacity and Rate Performance of Nanostructured TiO2(B). Langmuir 2012, 28, 28972903.

30. Ong, G. K.; Williams, T. E.; Singh, A.; Schaible, E.; Helms, B. A.; Milliron, D. J., Ordering in Polymer Micelle-Directed Assemblies of Colloidal Nanocrystals. Nano Lett. 2015, 15, 82408244.

31. Lui, G.; Li, G.; Wang, X. L.; Jiang, G. P.; Lin, E.; Fowler, M.; Yu, A. P.; Chen, Z. W., Flexible, three-dimensional ordered macroporous $\mathrm{TiO} 2$ electrode with enhanced electrode electrolyte interaction in high-power Li-ion batteries. Nano Energy 2016, 24, 72-77.

32. Wang, Y.; Fedin, I.; Zhang, H.; Talapin, D. V., Direct optical lithography of functional inorganic nanomaterials. Science 2017, 357, 385-388.

33. Helms, B. A.; Williams, T. E.; Buonsanti, R.; Milliron, D. J., Colloidal Nanocrystal Frameworks. Adv. Mater. 2015, 27, 5820-5829.

34. De Roo, J.; De Keukeleere, K.; Hens, Z.; Van Driessche, I., From ligands to binding motifs and beyond; the enhanced versatility of nanocrystal surfaces. Dalton Trans. 2016, 45, 1327713283.

35. Mourdikoudis, S.; Liz-Marzan, L. M., Oleylamine in Nanoparticle Synthesis. Chem. Mat. 2013, 25, 1465-1476.

36. Yin, Y.; Alivisatos, A. P., Colloidal nanocrystal synthesis and the organic-inorganic interface. Nature 2005, 437, 664-670.

37. Rechberger, F.; Heiligtag, F. J.; Suess, M. J.; Niederberger, M., Assembly of BaTiO3 Nanocrystals into Macroscopic Aerogel Monoliths with High Surface Area. Angew. Chem.-Int. Edit. 2014, 53, 6823-6826.

38. Grote, C.; Cheema, T. A.; Garnweitner, G., Comparative Study of Ligand Binding during the Postsynthetic Stabilization of Metal Oxide Nanoparticles. Langmuir 2012, 28, 1439514404.

39. De Roo, J.; Van den Broeck, F.; De Keukeleere, K.; Martins, J. C.; Van Driessche, I.; Hens, Z., Unravelling the Surface Chemistry of Metal Oxide Nanocrystals, the Role of Acids and Bases. J. Am. Chem. Soc. 2014, 136, 9650-9657.

40. De Roo, J.; Van Driessche, I.; Martins, J. C.; Hens, Z., Colloidal metal oxide nanocrystal catalysis by sustained chemically driven ligand displacement. Nat Mater 2016, 15, 517-521.

41. Anderson, N. C.; Hendricks, M. P.; Choi, J. J.; Owen, J. S., Ligand Exchange and the Stoichiometry of Metal Chalcogenide Nanocrystals: Spectroscopic Observation of Facile Metal-Carboxylate Displacement and Binding. J. Am. Chem. Soc. 2013, 135, 18536-18548.

42. Siramdas, R.; McLaurin, E. J., InP Nanocrystals with Color-Tunable Luminescence by Microwave-Assisted lonic-Liquid Etching. Chem. Mat. 2017, 29, 2101-2109.

43. Lan, X. Z.; Voznyy, O.; de Arquer, F. P. G.; Liu, M. X.; Xu, J. X.; Proppe, A. H.; Walters, G.; Fan, F. J.; Tan, H. R.; Liu, M.; Yang, Z. Y.; Hoogland, S.; Sargent, E. H., 10.6\% Certified Colloidal Quantum Dot Solar Cells via Solvent Polarity-Engineered Halide Passivation. Nano Lett. 2016, $16,4630-4634$.

44. Llordes, A.; Garcia, G.; Gazquez, J.; Milliron, D. J., Tunable near-infrared and visiblelight transmittance in nanocrystal-in-glass composites. Nature 2013, 500, 323-326. 
45. Ibanez, M.; Korkosz, R. J.; Luo, Z. S.; Riba, P.; Cadavid, D.; Ortega, S.; Cabot, A.; Kanatzidis, M. G., Electron Doping in Bottom-Up Engineered Thermoelectric Nanomaterials through HCl-Mediated Ligand Displacement. J. Am. Chem. Soc. 2015, 137, 4046-4049.

46. Xiao, L. L.; Lin, D. K. J.; Bai, F. S., Constructing Definitive Screening Designs Using Conference Matrices. J. Qual. Technol. 2012, 44, 2-8.

47. Jones, B.; Nachtsheim, C. J., A Class of Three-Level Designs for Definitive Screening in the Presence of Second-Order Effects. J. Qual. Technol. 2011, 43, 1-15.

48. Doris, S. E.; Lynch, J. J.; Li, C. Y.; Wills, A. W.; Urban, J. J.; Helms, B. A., Mechanistic Insight into the Formation of Cationic Naked Nanocrystals Generated under Equilibrium Control. J. Am. Chem. Soc. 2014, 136, 15702-15710.

49. Tomita, K.; Petrykin, V.; Kobayashi, M.; Shiro, M.; Yoshimura, M.; Kakihana, M., A water-soluble titanium complex for the selective synthesis of nanocrystalline brookite, rutile, and anatase by a hydrothermal method. Angew. Chem.-Int. Edit. 2006, 45, 2378-2381.

50. De Keukeleere, K.; De Roo, J.; Lommens, P.; Martins, J. C.; Van der Voort, P.; Van Driessche, I., Fast and Tunable Synthesis of ZrO2 Nanocrystals: Mechanistic Insights into Precursor Dependence. Inorg. Chem. 2015, 54, 3469-3476.

51. De Roo, J.; Coucke, S.; Rijckaert, H.; De Keukeleere, K.; Sinnaeve, D.; Hens, Z.; Martins, J. C.; Van Driessche, I., Amino Acid-Based Stabilization of Oxide Nanocrystals in Polar Media: From Insight in Ligand Exchange to Solution H-1 NMR Probing of Short-Chained Adsorbates. Langmuir 2016, 32, 1962-1970.

52. Bullard, J. W.; Cima, M. J., Orientation dependence of the isoelectric point of TiO2 (rutile) surfaces. Langmuir 2006, 22, 10264-10271.

53. Diebold, U., The surface science of titanium dioxide. Surf. Sci. Rep. 2003, 48, 53-229.

54. Liu, Y.; García, G.; Ortega, S.; Cadavid, D.; Palacios, P.; Lu, J.; Ibáñez, M.; Xi, L.; De Roo, J.; López, A. M.; Martí-Sánchez, S.; Cabezas, I.; Mata, M. d. I.; Luo, Z.; Dun, C.; Dobrozhan, O.; Carroll, D. L.; Zhang, W.; Martins, J.; Kovalenko, M. V.; Arbiol, J.; Noriega, G.; Song, J.; Wahnón, P.; Cabot, A., Solution-based synthesis and processing of Sn- and Bi-doped Cu3SbSe4 nanocrystals, nanomaterials and ring-shaped thermoelectric generators. J. Mater. Chem. A 2017, 5, 2592-2602.

55. Zhou, Y.; Buhro, W. E., Reversible Exchange of L-Type and Bound-lon-Pair X-Type Ligation on Cadmium Selenide Quantum Belts. J. Am. Chem. Soc. 2017, 139, 12887-12890.

56. Chen, P. E.; Anderson, N. C.; Norman, Z. M.; Owen, J. S., Tight Binding of Carboxylate, Phosphonate, and Carbamate Anions to Stoichiometric CdSe Nanocrystals. J. Am. Chem. Soc. 2017, 139, 3227-3236.

57. De Roo, J.; Ibanez, M.; Geiregat, P.; Nedelcu, G.; Walravens, W.; Maes, J.; Martins, J. C.; Van Driessche, I.; Koyalenko, M. V.; Hens, Z., Highly Dynamic Ligand Binding and Light Absorption Coefficient of Cesium Lead Bromide Perovskite Nanocrystals. ACS Nano 2016, 10, 2071-2081.

58. Berestok, T.; Guardia, P.; Blanco, J.; Nafria, R.; Torruella, P.; Lopez-Conesa, L.; Estrade, S.; Ibanez, M.; de Roo, J.; Luo, Z.; Cadavid, D.; Martins, J. C.; Kovalenko, M. V.; Peiro, F.; Cabot, A., Tuning Branching in Ceria Nanocrystals. Chem. Mat. 2017, 29, 4418-4424.

59. Dong, A. G.; Ye, X. C.; Chen, J.; Kang, Y. J.; Gordon, T.; Kikkawa, J. M.; Murray, C. B., A Generalized Ligand-Exchange Strategy Enabling Sequential Surface Functionalization of Colloidal Nanocrystals. J. Am. Chem. Soc. 2011, 133, 998-1006.

60. Rosen, E. L.; Buonsanti, R.; Llordes, A.; Sawvel, A. M.; Milliron, D. J.; Helms, B. A., Exceptionally Mild Reactive Stripping of Native Ligands from Nanocrystal Surfaces by Using Meerwein's Salt. Angew. Chem.-Int. Edit. 2012, 51, 684-689. 
61. De Roo, J.; Justo, Y.; De Keukeleere, K.; Van den Broeck, F.; Martins, J. C.; Van Driessche, I.; Hens, Z., Carboxylic-Acid-Passivated Metal Oxide Nanocrystals: Ligand Exchange Characteristics of a New Binding Motif. Angew. Chem.-Int. Edit. 2015, 54, 6488-6491.

62. De Roo, J.; De Keukeleere, K.; Feys, J.; Lommens, P.; Hens, Z.; Van Driessche, I., Fast, microwave-assisted synthesis of monodisperse HfO2 nanoparticles. J. Nanopart. Res. 2013, $15,1778-1781$.

63. Kosmulski, M., The significance of the difference in the point of zero charge between rutile and anatase. Adv. Colloid Interface Sci. 2002, 99, 255-264.

64. De Keukeleere, K.; Coucke, S.; De Canck, E.; Van Der Voort, P.; Delpech, F.; Coppel, Y.; Hens, Z.; Van Driessche, I.; Owen, J. S.; De Roo, J., Stabilization of Colloidal Ti, Zr, and Hf Oxide Nanocrystals by Protonated Tri-n-octylphosphine Oxide (TOPO) and Its Decomposition Products. Chem. Mat. 2017, 29, 10233-10242. 
TOC graphic
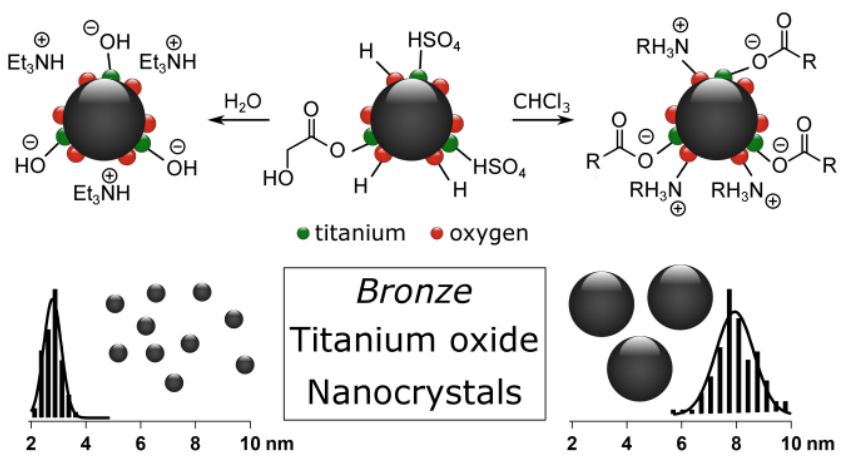